\title{
Kulturelle Dimensionen des professionellen Handelns - eine empirische Rekonstruktion der Orientierungen von Lehramtsstudierenden
}

\author{
Susanne Timm (iD
}

Eingegangen: 4. März 2020 / Überarbeitet: 21. Oktober 2020 / Angenommen: 11. Januar 2021 / Online publiziert: 27. Januar 2021

(C) Der/die Autor(en) 2021

Zusammenfassung Lehrkräfte sind als Kulturgeprägte kulturprägend tätig. Weit jenseits dessen, dass sie explizit über die Vermittlung kulturbezogener Wissensbestände und kultureller Techniken in Kultur einführen, ist ihr Handeln implizit kulturbezogenes Vermittlungshandeln, das habituell geprägt ist. In dieser Studie steht die Frage im Mittelpunkt, welches implizite Wissen für die kulturelle Dimension professionellen Handelns für Lehramtsstudierende handlungsleitend ist. Angesichts der langfristigen Ausprägungen habitueller Konstitutionen und angesichts von Anforderungen an die Professionalität von Lehrkräften in einer kulturell diversen Gesellschaft wurde untersucht, welche Orientierungen hinsichtlich von Kulturalität und damit einhergehenden Vorstellungen zu den impliziten Anforderungen an Lehren Lehramtsstudierende im Studium ausprägen. Diese Frage wurde im rekonstruktiven Paradigma in einer qualitativen Studie mit Hilfe von Gruppendiskussionen und einer abduktiven Typenbildung bearbeitet. An mehreren der idealtypisch gewonnen Orientierungstypen zeigt sich, dass dominante Deutungen der Mehrheitskultur handlungsleitend sind und zumeist in unreflektierten Wiederholungen zur Geltung kommen. Vor diesem Hintergrund werden Anregungen und Herausforderung für die Ausbildung von Lehrerinnen und Lehrern sowie für die weitere Lehrerbildungsforschung formuliert.

Schlüsselwörter Lehreramtsstudierende · Orientierungen · Kulturelle Bildung

Dr. S. Timm $(\bowtie)$

Lehrstuhl für Allgemeine Pädagogik, Otto-Friedrich-Universität Bamberg,

Luisenstraße 5, 96047 Bamberg, Deutschland

E-Mail: susanne.timm@uni-bamberg.de 


\title{
Acting professional in a cultural way-an empirical reconstruction of orientations of student teachers
}

\begin{abstract}
Teachers are transmitting culture as culturally influenced persons. Far beyond the fact that they explicitly introduce into culture through the teaching of culture-related knowledge and cultural techniques, their practices are implicitly culture-related as they are shaped by their habitus. This study focuses on the implicit knowledge for the cultural dimension of professional action which is guiding action for student teachers. Due to the long-term characteristics of habitual constitutions and in view of the demands on the professionalism of teachers in a culturally diverse society, the study examines which orientations with regard to culturality and the associated ideas on the implicit demands on teaching are shaped by student teachers during their studies. This question was addressed in a qualitative study in the reconstructive paradigm using group discussions and abductive type formation. Several of the ideal-typical orientation types show that dominant interpretations of the majority culture guide action and are usually expressed in unreflected repetitions. Against this background, suggestions and challenges for teacher training and further teacher education research are formulated.
\end{abstract}

Keywords Cultural education · Orientations · Teacher Students

\section{Einleitung}

Welche impliziten Wissensbestände und habituellen Prägungen sind für Lehramtsstudierende im Feld kulturellen Professionshandeln leitend? Wie reflektieren sie ihr kulturprägendes professionelles Handeln, das sie als Kulturgeprägte ausführen? Mit diesen Fragen soll ein Aspekt des professionellen Werdens von Lehramtsstudierenden, der sukzessive Prozess der Erfahrungsaufschichtung und Wissensaneignung im Zuge einer habitueller Transformation, in den Blick genommen werden, der als impliziter fachübergreifend für Lehrerhandeln bedeutsam und empirisch wenig beforscht ist. Jenseits dessen, dass Lehrkräfte explizit über die Vermittlung kultureller Wissensbestände und kultureller Techniken in Kultur einführen, handeln sie implizit kulturtradierend und kulturprägend. Sie exemplifizieren Lesarten von Welt, sie offerieren Deutungsangebote und formen mit ihren Werthaltungen den Interpretationsrahmen von Welt, sie repräsentieren und verkörpern kulturelle Ordnungen. Kurz: Sie sind Träger und Performierende der Kultur, in die Schule die nachwachsende Generation einführt.

Die handlungsleitenden Orientierungen angehender Lehrkräfte standen im Mittelpunkt einer Studie im Rahmen des BMBF-geförderten Projektes „Kulturelle Lehrerbildung - KulturLeBi. Grundlagen einer kulturbezogenen Lehrerbildung“ (FKZ 01JK1602). Gefragt wurde danach, welches implizite Wissen für die kulturelle Dimension professionellen Handelns für Lehramtsstudierende handlungsleitend ist. Ziel war es, auf der Grundlage einer qualitativ-rekonstruktiven Studie dieses handlungsleitende Wissen zu rekonstruieren, empirisch fundiert zu beschreiben und Konsequenzen für die Lehrerbildung und die weitere Forschung zu formulieren. 
Im Nachfolgenden werden zunächst die Fragestellung kulturtheoretisch geschärft, schul- und professionstheoretisch angeschlossen, der Forschungsstand skizziert, Desiderate markiert. Im zweiten Abschnitt werden das Vorgehen mit Erhebungen in Gruppendiskussionen (Bohnsack et al. 2010) und deren dokumentarischen Interpretation (Bohnsack 2006) sowie der methodische Zugriff näher erläutert. Die Ergebnisse der rekonstruktiven Untersuchung werden im dritten Teil dargestellt. Im Zentrum steht dabei die Typologie der Orientierungen für das Feld kulturellen Professionshandelns bei Lehramtsstudierenden. Dieses Ergebnis wird im abschließenden vierten Teil im Fokus auf die Varianz im Umgang mit kulturellen Deutungen zusammengefasst und im Hinblick auf die Professionalisierung für den Lehrberuf diskutiert. Ein zentraler Befund, dass im kulturellen Horizont der Studierenden die habituell verankerten dominanten Deutungen der Mehrheitskultur zumeist in unreflektierten Wiederholungen zur Geltung gebracht werden, gibt dafür den Anstoß, Lehrkräftebildung prägnanter mit kultureller Reflexivität als Professionalisierungsperspektive $\mathrm{zu}$ verbinden.

\section{Das Handlungsfeld Schule in seinen Kulturbezügen und seiner Bedeutung für die Lehrkräftebildung}

\subsection{Theoretische Anschlüsse einer Lehrerbildungsforschung im Feld kulturellen Professionshandeln}

Die Studie gründet auf ein Verständnis von Kultur als ordnungsbildendem Sinnzusammenhang. In der Referenz auf Clifford Geertz' semiotischen Kulturbegriff ist Kultur als Sinn- und Bedeutungsbezug alltäglicher und sozialer Handlungen konstituiert und der Mensch in Kultur als das „selbstgesponnene Bedeutungsgewebe“ verstrickt (vgl. Geertz 2016 [1973], S. 5). Kultur äußert sich in symbolischen Verweisungszusammenhängen, die zwischen individuellen Interpretationen und überindividuellen Referenzhorizonten oszillieren. Kultur offeriert Anschlüsse für den Einzelnen, sie muss gedeutet und interpretiert werden. Noch in der Enkulturation verbleiben Kulturproduktion und -reproduktion in einer reziproken Beziehung. Ein solches Kulturverständnis hat weitreichende Konsequenzen für die Konzeptionierung von Bildung, wie sie von Rosenberg (2016) unter Rekurs auf Reckwitz und Nohl im Hinblick auf die Komplexität und Mehrdimensionalität im Spannungsfeld von Sozialisation, Zugehörigkeit und Bildung entfaltet.

Kulturelle Bildung wird in dem hier vorgestellten Forschungszusammenhang zunächst als Selbstbildung im Modus des Erschließens von Sinnzusammenhängen und der Re-Interpretation von Welt verstanden, die als kulturelle Objektivationen und sedimentierte Materialisierungen, als Normen und Werthorizonte sowie als bedeutungstragende Chiffren dem Einzelnen entgegentreten. Neben hermeneutischen Kompetenzen als bildungstheoretische Antwort auf die Unabgeschlossenheit von Bedeutungen (vgl. Koerrenz 2014) ist kulturelle Bildung in einer kulturwissenschaftlichen Perspektive auf ihre gesellschaftliche Dimension dann auszuweiten, wie Krenz-Dewe (vgl. 2017) in seiner Fassung kultureller Bildung als kritische unmissverständlich zum Ausdruck bringt. So sei „die Einsicht in die Veränderbarkeit von 
Subjektpositionen und Differenzordnungen und die Wahrnehmung und Erfahrung dieser Veränderbarkeit als eine normative Bahnung von kulturellen Bildungsangeboten zu verstehen." (Krenz-Dewe 2017, S. 423). Explizite Momente, wie sie sich in den vielfältigen Formen Kultureller Bildung und in deren Reflexionen äußern (vgl. Bockhorst et al. 2012; Fink et al. 2012; Liebau 2013; Zacharias 2013), sind in der hier vorgeschlagenen Perspektive Ausschnitte einer kulturellen Bildung, die als implizit sich entfaltender Prozess des Erschließens und Sichaneignens, des Aushandelns und Modifizierens von Bedeutungsdimensionen verstanden wird. Um begrifflich die Mehrdimensionalität und auch den impliziten Charakter so verstandener kultureller Bildungsprozesse aufzufangen, schlagen Bauer et al. vor, von ,kulturbezogener Bildung“ zu sprechen (Bauer et al. 2018, S. 15). Ihrer Abgrenzung zur Bezeichnung intendierter Vermittlungsprozesse kann sich hier angeschlossen werden. In der Betonung auf das kulturell Implizite jeglicher Prozesse im Kontext der Bildung des Subjektes wird aber der Terminus der kulturellen Bildung beibehalten.

Das Individuum bedarf der Möglichkeit des Verstehens, des Interpretierens und des Reformulierens der vorgängigen und pluralen Weltdeutungen, der Fähigkeiten des En- und Decodierens (vgl. Hall 1973), um in dem Netzwerk von Sinnzusammenhängen und ihren sozialen Bezügen handeln zu können. Schule ist als gesellschaftliche Institution der Ort, an welchem der Einzelne jenseits der Kulturbezüge familiärer Kontexte an verschiedene Weltzugänge herangeführt und in die Dynamiken der Mehrdeutigkeit gestellt wird. Sie fungiert in dieser Hinsicht als Ort der Enkulturation (vgl. Fend 2006) und ist grundlegend für das Einüben des Verstehens der Bedeutungsdimensionen des Sozialen wie für das Vermögen, sich an sinnbezogenen Aushandlungsprozessen zu beteiligten. Diese strukturtheoretisch gefasste Funktion von Schule als ein Ort der Enkulturation ist an sich spannungsreich, als Schule in Kultur als gesellschaftlichen Plural von Bedeutungen nur über die Singularität der je spezifischen Schulkultur (vgl. Göhlich 2007) einführen kann. Lehrkräfte handeln dabei unter widersprüchlichen Bedingungen, als sie als habituell kulturgeprägte (vgl. Helsper 2018) in einem kulturell formierten Kontext (Schule) in die pluralisierte Gesellschaft vielfältiger Kulturen einführen. Die habituellen Prägungen der Lehrkräfte werden angesichts gesellschaftlicher Diversifizierung, den zugleich wirksamen Individualisierungsprozessen (vgl. Reckwitz 2017) und den damit gesteigerten Orientierungsherausforderungen (vgl. Scheunpflug 2016), wie sie auch aus der Dynamisierung von Differenz- und Differenzierungsprozessen resultieren (vgl. Hirschauer 2014), besonders bedeutsam, zumal die kulturellen Dimensionen des Handelns von Lehrkräften, die durch sie erfahrbar werdenden kulturellen Repräsentationen jenseits curricularer Inhalte, zumeist im Impliziten verbleiben.

Angesichts dieser komplexen Rahmung des Handelns von Lehrkräften in seinen kulturellen Dimensionen liegt es nahe, dieses Handeln unter einer Professionalitätsperspektive genauer zu betrachten und auch seine Voraussetzungen zu klären. Die habituelle Bindung bedeutet, dass das steuernde implizite Wissen über den Lebenslauf und seine verschiedenen Erfahrungsräume aufgeschichtet wird. Es wird im Handeln erworben, und verschiebt sich über die verschiedenen Erfahrungs- und Handlungsräume lediglich graduell. Lehrerbildung nimmt insofern für die Grundlegung des beruflichen Habitus (vgl. Kramer 2015) eine Schlüsselposition ein, als Lehrkräfte in dieser Phase im Übergang zwischen persönlichem sowie schulischem 
Werdegang und der Ausbildung eines beruflichen Habitus stehen. Mit den Erfahrungen im Studium werden im Anschluss an die habituellen Vorprägungen kollektive Orientierungen ausgeprägt, die auch für die kulturelle Dimensionen des professionellen Handelns leitend werden (vgl. Helsper 2019). Angesichts der impliziten Einträge in das Handeln der Lehrkräfte gewinnt Lehrerbildung auch dadurch eine besondere Bedeutung, als in ihrem Kontext eine Aufklärung über die Tatsachen der eigenen Vorprägungen und die dilemmatische Struktur kulturellen Professionshandeln im schulischen Kontext, die aus der individuell vorgängigen Kulturgeprägtheit und einer schulkulturellen Rahmung resultieren, erfolgen kann.

\subsection{Forschung zu professionellem Handeln im kulturellen Feld}

Das doppelte kulturelle Orientierungsparadox zwischen Schulkultur und individuellen habituellen Vorprägungen der Lehrkräfte sowie zwischen dem Singular einer in Schule präsentierten Kultur und dem Plural kultureller Orientierungsoptionen schlägt sich bislang in der Forschung nur partiell in unterschiedlichen Diskurssträngen nieder:

1. Ein Bearbeitungsfokus liegt im Themenfeld der kulturellen Pluralität hinsichtlich des Migrationsaspektes und mit dem besonderen Augenmerk auf Lehrkräfte (Mecheril 2008; Lanfranchi 2008). Im internationalen Kontext wird dieses Themenfeld gegenwärtig mit einem Schwerpunkt auf cultural responsiveness bearbeitet (Vavrus 2008; Lehtomäki et al. 2017).

2. In einem weiteren Forschungsspektrum wird der Zusammenhang von Schule und Kultur vor allem auf ästhetische Dimensionen fokussiert (Fuchs und Braun 2016; Liebau 2019). Kennzeichnend ist hier, dass Schule als Kulturort insbesondere durch ästhetische Praxen in kulturelle Diversität einführen kann. Lehrkräfte werden zwar als Kultur Performierende benannt, die Forschungsinteressen sind aber deutlicher schultheoretisch ausgerichtet und auf aisthetische Praxen zentriert.

3. Schließlich finden im fachdidaktischen und bildungswissenschaftlichen Forschungsspektrum Studien zum Zusammenhang zwischen eigenen Kulturprägungen, Bildungserfahrungen und professionellen Orientierungen Berücksichtigung, insbesondere in der Fokussierung musischer Fächer und im Zusammenhang sportlicher und damit explizit körperbezogener Erfahrungshaushalte (u. a. Dwyer 2016; Meister 2018).

4. In einzelnen Studien werden die Einstellungen von Lehramtsstudierenden im Themenfeld analysiert (Schieferdecker 2015), andere fokussieren durchaus die Problematik der habituellen Situiertheit ihres Handelns (Kramer und Helsper 2010; Kramer und Pallesen 2019). Kultur wird im Kontext von Schule dann zumeist auf Mechanismen der Reproduktion von Ungleichheit bezogen, ein Zusammenhang, der die Bedeutung kultureller Vorprägungen von Lehrkräften als gesellschaftsrelevante unterstreicht. Das Augenmerk auf Lehrkräfte blendet Lehramtsstudierende, folglich auch die Universität als Bearbeitungskontext vorgängiger Kulturprägungen, eher aus.

Studien zur habituellen Grundlegung kulturellen Professionshandeln jenseits fachspezifischer Zuschnitte und jenseits von Akzentuierungen auf Schule in der 
Migrationsgesellschaft oder berufsbiographischer Perspektivierungen finden sich trotz dieser Fülle also nicht. Insbesondere fehlen übergreifende Untersuchungen zu den kulturbezogenen Orientierungen Lehramtsstudierender.

\subsection{Präzisierung der Fragestellung}

An diese Desiderate wird nun angeknüpft und die Frage gestellt, mit welchen Orientierungen Lehramtsstudierende in das Handlungsfeld schulischer Enkulturation, das als Verwicklungszusammenhang umfassender Offerten von Bedeutungszusammenhängen mit der Selbstzuordnung der Nachwachsenden in Bedeutungswelten verstanden wird (vgl. Prange 1978), eintreten. Welche Konstruktionen von Kultur lassen sich unter Lehramtsstudierenden auffinden? Welche Orientierungsaspekte leiten ihre Entwürfe professionellen Handelns in seinen kulturellen Dimensionen? Lassen sich Hinweise dafür auffinden, dass sich Lehramtsstudierende in ein Verhältnis zu ihrem eigenen kulturellen Horizont setzen und um dessen Möglichkeiten wie Limitationen im Umgang mit multiplen Sinnzusammenhängen wissen? In welcher Weise nähern sie sich den impliziten Anteilen ihres Professionshandeln und seinen kulturellen Bezügen an? Mit einer empirisch fundierten Beschreibung der handlungsleitenden Orientierungen angehender Lehrkräfte wird schließlich das Ziel verfolgt, Lehrerbildung als Transformationsanlass zwischen den kulturellen Horizonten des eigenen Werdens und den kulturellen Dimensionen des zukünftigen Professionshandelns zu fundieren.

\section{Die Annäherung an die Empirie: Die Rekonstruktion der Orientierungsrahmen}

Für die angestrebte Rekonstruktion der handlungsleitenden Orientierungen von Lehramtsstudierenden in Bezug auf die kulturellen Dimensionen ihres Professionshandelns wurden Daten in Gruppendiskussionen erhoben (Bohnsack et al. 2010) und mit der dokumentarischen Methode (Bohnsack 2006) ausgewertet.

\subsection{Methodologische Grundlegungen}

Orientierungen werden in der methodologischen Grundlegung der dokumentarischen Methode als implizite Wissenselemente verstanden, die das Handeln von Personen fundieren. Sie können als ,strukturierende Struktur beschrieben“werden, die in einem zirkulären Verständnis - analog zum Habitus - auch als strukturierte verstanden werden (Bohnsack 2006). Sie sind dem explizierbaren Wissen der Personen nicht zugänglich, können aber mittels rekonstruktiver Forschungsverfahren zur Sprache gebracht werden. Dies beruht auf der Annahme, dass das implizite bzw. atheoretische Wissen der Orientierungen nicht nur in kollektiven Praxen erworben wird, sondern auch in ihnen zur Geltung kommt. Diese performative Seite der Orientierungen ist die Grundlage für das gewählte Erhebungsverfahren in Gruppendiskussionen: Diskussionen, in denen über offene Gesprächsimpulse möglichst selbstläufige Diskussionsverläufe induziert werden, werden als geteilte Praxis der Gruppen auf- 
gefasst, in denen die geteilten Orientierungen der Beteiligten aktiviert werden. Zwei Aspekte finden beim weiteren Vorgehen besondere Berücksichtigung: Die Gruppendiskussionen verweisen als geteilte Praxis auf die jeweils geteilten Orientierungen, in denen sich das Orientierungsset der Einzelperson keinesfalls erschöpfen muss. Über einzelne Personen können demnach keine Aussagen getroffen werden, ebenso nicht für die Involviertheit der Untersuchten in andere Erfahrungsräume und den in ihnen erworbenen Orientierungen. Der zweite Aspekt bezieht sich auf die Relationen zwischen den aufgeworfenen Themen in den Gruppendiskussionen und den rekonstruierten Orientierungen: Während die inhaltliche Dimension, das Was der Gruppendiskussion, thematisch an das Forschungsinteresse anschließt, gilt für die Rekonstruktion der Orientierungen die Aufmerksamkeit dem Wie, dem Modus der Themenbearbeitung in den Diskussionen (Bohnsack 2013).

Das Interpretationsverfahren der transkribierten Gruppendiskussionen ist iterativ über verschiedene Ebenen des Datenmaterials in Vergleichsoperationen angelegt, zunächst innerhalb der Diskussionsverläufe der einzelnen Gruppen, dann diese übergreifend. Dadurch werden im Zuge des Forschungsprozesses die Vergleichshorizonte ausgedehnt, zunehmend wird auf Vergleichspunkte aus dem empirischen Material zurückgegriffen und werden dadurch die Vorannahmen der Untersuchenden ausgeschlossen. Schließlich werden die Rekonstruktionen in einem abduktiven Prozess in einen idealtypischen Typenbildungsprozess überführt, in der Nomenklatur der dokumentarischen Methode ist es die sinngenetische Typenbildung. ${ }^{1}$

\subsection{Das Design der Studie}

Im Rahmen der Studie wurden Daten in 28 Gruppendiskussionen mit insgesamt 112 Teilnehmenden an bayerischen Universitäten erhoben. Sie wurden aus Lehrveranstaltungen und extracurricularen Aktivitätszusammenhängen gewonnen, d.h. sie wiesen als Gruppe je einen geteilten Erfahrungsraum auf. Die Samplekonstituierung folgte in einem ersten Schritt theoretischen Vorüberlegungen, und dabei einer Verteilung über verschiedene Fachgruppen Lehramtsstudierender sowie bildungswissenschaftlicher Studienzusammenhänge hinweg. Einem Theoretical Sampling (Glaser und Strauss 1967) folgend, wurde nach ersten Interpretationen das Sample in weiteren Schritten um Gruppen aus Studienzusammenhängen jenseits des Lehramts und um geteilte Erfahrungen in außeruniversitären Aktivitäten ergänzt. Insgesamt setzte sich das Sample aus künstlerisch orientierten Fachgruppen (sieben Gruppen mit Fachperspektive Musik oder Bildender Kunst, 28 Teilnehmende, davon fünf Gruppen mit Lehramtsbezug und zwei kontrastierende Gruppen) und aus geisteswissenschaftlichen Fächern (acht Gruppen mit Fachperspektive Geschichte, Religion, Fremdsprachlicher Unterricht und Deutsch als Fremdsprache, 24 Teilnehmende), aus erziehungswissenschaftlichen Lernzusammenhängen (elf Gruppen, davon neun

\footnotetext{
${ }^{1}$ Das Verfahren der dokumentarische Methode sieht neben der sinngenetischen Typenbildung eine soziogenetische vor, ein Schritt, durch den über weitere Vergleichsoperationen die spezifischen Charakteristika der Erfahrungsräume, die sich mit bestimmten Orientierungsgehalten verknüpfen lassen, herausgearbeitet werden. In dieser Hinsicht lassen sich für die Untersuchung der Orientierungen von Lehramtsstudierenden Tendenzen äußern, eine fundierte soziogenetische Typenbildung ist gleichwohl nicht abgeschlossen.
} 
mit explizitem Kulturbezug, 52 Teilnehmende) und aus selbstorganisiert politischen Gruppen (zwei Gruppen aus inner- und außeruniversitären kulturpolitischen Initiativen, acht Teilnehmende) zusammen. Der Fokus auf den Fachzusammenhang in der Rekrutierung der Gruppen führte dazu, dass 21 von 28 Gruppen aus Lehramtsstudiengängen mit unterschiedlichen Schulperspektiven zusammengesetzt war.

In den Gruppendiskussionen wurden mit einem offenen Gesprächsimpuls zu den Erfahrungen im Lernzusammenhang und deren Bedeutungen für die Personen, das Studium und zukünftigen Handlungsperspektiven selbstläufige Diskussionen initiiert (Loos und Schäffer 2001), die aufgenommen, regelgeleitet transkribiert (Bohnsack 1999, S. 33 ff.) und zur weiteren Verarbeitung anonymisiert wurden. Die dokumentarische Interpretation erfolgte in den Schritten der formulierenden und der reflektierenden Interpretation und mit einer sich anschließenden Typenbildung. Geht es im ersten Interpretationsschritt darum, das theoretische und explizierbare Wissen, das in den Gruppendiskussionen zur Sprache kommt, zu bündeln, wird im zweiten die Diskursorganisation fokussiert. Über verschiedene Vergleichsschritte inner- und außerhalb der einzelnen Gruppendiskussionen konnte der Modus herausgearbeitet werden, in welchem die Themen von den Gruppen bearbeitet werden, wodurch die den Thematisierungen zugrundgelegten Orientierungen rekonstruiert wurden. Diese Schritte wurden wie die mittels Abduktion gewonnene, idealtypisch verdichtete sinngenetische Typenbildung getreu den Qualitätsanforderungen qualitativer Forschung kontinuierlich in zwei Interpretationsgruppen kommunikativ und konsensuell validiert $^{2}$ (zu den Standards qualitativ-rekonstruktiver Forschung Bohnsack 2005). Die resultierende Typologie der Orientierungen von Lehramtsstudierenden wird im Nachfolgenden erläutert.

\section{Die Typologie: Agenten und Passanten im Feld kulturellen Professionshandelns}

Alle Gruppen, die im Rahmen der Untersuchung erhoben wurden, konzentrieren sich in ihren Diskussionen auf die Frage, wie sie mit Dynamiken im kulturellen Netz von Bedeutungen umgehen oder umgehen könnten. Dieser Inhalt bildet den Kern bzw. in der Terminologie der dokumentarischen Methode die Basistypik. Veränderungsimpulse aus kulturellen Dynamiken werden in den Gruppen anhand unterschiedlicher Themen bearbeitet, die in den selbstläufigen Gruppendiskussionen ohne weitere Impulse der interviewenden Person aufgeworfen wurden. Vier im Material verankerte thematische Aspekte kristallisierten sich in den fallübergreifenden Vergleichsoperationen heraus. Es resultierte eine Matrix analytisch geschiedener thematischer Dimensionen, die eine erste Grundlage für die abduktiv gewonnene Typenbildung darstellt (Abschn. 4.1). Den Gegenstand des Vergleichs bildeten dann die Bearbei-

\footnotetext{
2 Ich danke herzlich Caroline Rau, Annette Scheunpflug, Dorothea Taube, die in insgesamt 25 Sitzungen die Interpretationen (17 IG 1 und 8 IG 2) validierten sowie Jana Costa, Barbara Drechsel, Claudia Kühn, Konstantin Lindner und Sabine Vogt, die in insgesamt zwölf mehrstündigen Sitzungen zur Reflexion des Forschungsprozesses beigetragen haben.
} 
tungsweisen der Themen, die unterschiedlichen Modi. Sie bilden die Grundlage für die Ausdifferenzierung der Typologie in fünf Orientierungstypen (Abschn. 4.2).

\subsection{Themen der fallübergreifenden Vergleiche}

Der Forschungslogik folgend ist der Beschreibung der Typen die der thematischen Vergleichsdimensionen vorangestellt. Sie gibt einen Einblick in die Themen, die in den Gruppendiskussionen aufgrund des offenen Impulses als inhaltlich relevante aufgeworfen wurden.

1. In den Konstruktionen von Welt im kulturellen Feld von Sinn und Bedeutung sind die inhaltlichen Bestimmungen der jeweiligen Kulturverständnisse erfasst. Es konnten zwei Schwerpunkte herausgearbeitet werden. Ein erster bildet Kultur als Differenzmatrix der Zugehörigkeit. Es ist eine Konstruktion von Kultur, in der jedem Menschen Kultur zugesprochen wird, und über die Menschen in soziale Zusammenhänge eingebettet, aber auch voneinander unterschieden sind. Der zweite Schwerpunkt liegt deutlich erkennbar im kulturell-ästhetischen Handlungsspektrum wie zu musizieren oder an Mappen zu arbeiten.

2. In der thematischen Vergleichsdimension kultureller Praxen der Produktion und Reproduktion lassen sich die Handlungen fassen, die in den Gruppendiskussionen zur Sprache kommen. Sie reichen vom Üben als routinierter Praxis im hochkulturellen Spektrum über das Sich-Hineinhören und -denken in Ansprachen zum Reformationsjubiläum bis hin zum Reisen und Kennenlernen als fremd wahrgenommener kultureller Zusammenhänge. Abstrahierter lassen sich die Aktivitäten auch im Hinblick darauf beschreiben, in welchem Maß sie als reproduzierend oder produzierend entworfen sind.

3. In der thematischen Perspektive auf die Relationierungen von Selbst mit Gemeinschaft und Welt und deren Professionsbezug werden die Verortungen und Selbstpositionierungen in gemeinschaftlichen und gesellschaftlichen Bezügen in den Blick genommen, und erste Ausblicke auf das Professionsverständnis erkennbar. Erneut spielt die Frage der Zugehörigkeit eine Rolle, nun aber in ihrem Bezug auf das Selbst: Wo gehöre ich hin? Welche Position nehme ich ein? Auch hierfür sind zwei Schwerpunkte auffindbar. In einem ersten wird das Selbst in einem vorgängigen und hermetisch-statischen Gemeinschaftsbezug als einem kollektiven Selbst angehörig lokalisiert. Ein zweiter Schwerpunkt positioniert das durchaus auch gemeinschaftlich gebundene Selbst dagegen prädominant in seinem Gesellschaftsbezug. Der Professionsbezug lässt sich dadurch in diesem Zusammenhang auffinden, als das Selbst daran anknüpfend auch seine berufliche Rolle im Spannungsfeld von Gemeinschaft und Gesellschaft verortet.

4. Eine letzte thematische Vergleichsdimension nimmt epistemische Rahmungen professionellen Handelns im Kontext kultureller Reproduktion und Produktion in den Blick und führt zu den Grundlegungen einer kulturbezogenen Didaktik. Hier liegt der Fokus auf den Wissenskonstruktionen, die den Entwürfen professionellen Handelns zugrunde liegen. Neben solchen der unveränderlichen Geltung tradierter Episteme und Ordnungen, treten epistemische Konstruktionen, in die die Konstruktivität und Kontingenz tradierter Bedeutungsembleme einfließen. 
Auf ihrer Grundlage können didaktische Strategien entfaltet werden, die Räume für partizipative Re-Kreationen im professionellen Handeln eröffnen.

\subsection{Typen der Orientierungen im Feld kulturellen Professionshandelns}

Im Zuge der Typenbildung wurden die unterschiedlichen Bearbeitungsweisen der Themen verglichen, das heißt auch zunehmend von der thematischen Dimension abstrahiert. So konnten sukzessive die Typen herausgearbeitet und voneinander abgegrenzt werden. Es sind Idealtypen, in denen die einzelnen Gruppen nicht aufgehen, geschweige denn einzelne Personen. Alle fünf Typen sind als Agenten bzw. Passanten bezeichnet. Hierin äußert sich, dass sie alle Akteure im Feld kulturellen Professionshandeln sind, allerdings in unterschiedlichen Grundlegungen sowie mit unterscheidbarem Ausmaß an Explizität.

\subsubsection{Typ I: Orientierung an unidirektionaler Weitergabe als Einbindung in kulturelle Zugehörigkeitsordnungen - Agenten des Verweilens im kulturellen Status Quo}

Für diesen Orientierungstyp ist ein Entwurf von Kultur kennzeichnend, der auf eine anthropologisch fundierte Totalität von Kultur rekurriert: jeder Mensch hat Kultur und Anteil an einer Kultur. Charakterisiert ist dieser Bezug auf ein anthropologisches Verständnis durch den Bezug auf essenzialisierte und essenzialisierende intern homogene Kollektivsubjekte. Mit diesem Kulturkonzept ist die Welt als dauerhaft geordnete Sinnordnung entworfen, deren Strukturierung durch Differenzsetzungen eine besondere Betonung erfährt.

In dieser Passage der Gruppe Tarantel (siehe Abb. 1) ist die Orientierung an einer ethnisch-kulturell fundierten Logik der Differenzsetzung zu erkennen, die mit der Verortung des Selbst in seinen Gemeinschaftsbezügen einhergeht. Sie zeigt sich in wiederholten Bezugnahmen auf Erfahrungen in ausländischen Kontexten, ob als touristische oder aus Anlass eines Auslandssemesters. Die hohe Bedeutung, die Differenzen zugerechnet wird, wiederholt sich im Positivismus kategorisierter Fachbezüge für die eigene Profession. Auch hier liegt eine differenzorientierte Ordnung vor, der ein ontologischer Status zugerechnet wird. Grundlegend wird Kultur ein impliziter Charakter zugeschrieben, der der Reflexion und Verhandelbarkeit, und damit einer Variation nicht zugänglich ist. Das Selbst ist dann als Objekt der Sozialisation und als unhintergehbar Zugehöriges zu einem intern homogenen Kultursubjekt gerahmt. Ähnliche Orientierungen fanden sich im Typ II, den Agenten nahräumlicher Erfahrung und in abgeschwächter Form im Typ V, den Passanten ratloser Problematisierung.

Für die kulturbezogenen Praxen der Produktion und Reproduktion ist die Vorstellung von kultureller Produktion als erhaltende Reproduktion routinierter Handlungen tragend, die in der Sozialisation eingeübt und aus dem gegebenen Handlungsreservoir übernommen werden. Diese Praxen mit vornehmlich reproduzierendem Charakter münden in eine Affirmation kanonischer Kulturelemente zur Stabilisierung vorfindlicher Sinn- und Zugehörigkeitsordnungen. 


\begin{tabular}{|c|c|c|}
\hline 401 & Bw & L ○Ja des \\
\hline 402 & & stimmt das ist schon lustig $^{\circ}($.$) ich mein ich find- ich find auch grad wenn man im Ausland is$ \\
\hline 403 & Aw & ᄂ Jaa; \\
\hline 404 & $\mathrm{Bw}$ & oder wenn man halt eben auch Sprachen studiert und da halt auch reingeht in diese Kultur äh \\
\hline 405 & & alles, sieht man halt auch selber, den eignen Kulturbereich viel besser und was man was man \\
\hline 406 & Cw & L Jaaa; \\
\hline 407 & Aw & L ( \\
\hline 408 & Bw & hat weil man (1) deutsch is, also des is halt so das hab ich mir echt gedacht in den in de- ich \\
\hline 409 & Cw & L Jaaa; \\
\hline 410 & Bw & war ja in verschiedenen Ländern; und verschiedenen Zeiten und aber; das war eigentlich immer \\
\hline 411 & & so die Essenz die rausgebracht hab, gar nich so sehr dass man das andre Land so kenngelernt \\
\hline 412 & & hat schon auch aber am eigentlich am meisten sich selber; und halt sich selber auch so (.) \\
\hline 413 & Cw & L Sich selber ja; \\
\hline 414 & Bw & ja das is echt deutsch was ich da habe; oder das vermiss ich auch wirklich also so diese; (1) ja? \\
\hline 415 & Cw & L Dass man sich dessen mal bewusst wird ja; \\
\hline
\end{tabular}

Abb. 1 Transkriptausschnitt Gruppe Tarantel

In seiner Relationierung des Selbst zur Gemeinschaft geht dieses in ihr auf, zu Gesellschaft verschwindet seine Kontur in der Konstruktion des Selbst als Normerfüllender externer Instanzen. Dies schlägt sich darin nieder, dass zunächst das eigene Lernen als Wissensaneignung ohne Bezug zum Selbst entworfen ist. Lernen wird als Erfüllung von Normen gerahmt und als Erweiterung angehäufter Wissensbestände verstanden. Die Verantwortung für dieses Lernen wird dann den institutionellen Vorgaben zugeschrieben und bei der Universität angesiedelt. Das Selbst bleibt dagegen fremd gegenüber der Universität als Ort der Wissensproduktion.

Im Hinblick auf die epistemische Rahmung professionellen Handelns im Kontext kultureller (Re-)Produktion zeigt sich für Typ I ein dominantes Verständnis von Vermittlung institutionell normierter Wissensbestände, mit einem Fokus auf die unidirektionale Wissensvermittlung.

Kulturbezogenes professionelles Handeln ist für Typ I als unhintergehbarer Enkulturationsprozess in intern homogene Kollektivsubjekte und als unidirektionale Weitergabe kulturell überlieferter Wissensgegenstände gefasst, als reproduzierende Praxis, deren Effekt in der Affirmation bestehender Ordnungsverhältnisse liegt. Die dominante Perspektive auf das Selbst als unhintergehbar in vorfindliche Gemeinschaftsbezüge Eingebundenes führt Lehramtsstudierende als überwiegend Angehörige der Mehrheitskulturen zur impliziten, aber machtvollen Replikation der homogen entworfenen Mehrheitskultur, mit dem Effekt eines nicht unproblematischen Verweilens im vermeintlichen kulturellen Status Quo.

\subsubsection{Typ II: Orientierung an Anleitung zum Lernen in exkludierenden Praxisgemeinschaften - Agenten nahräumlicher Erfahrungen}

Für diesen Typ ist ein sektorales und innerhalb dessen ein konventionelles Kulturverständnis grundlegend, das mit einer Ausrichtung auf statisch und unveränderliche Ordnungsbildung durch tradierte Normen amalgiert. Kennzeichnend ist ein Fokus auf kanonisch gekennzeichnete hochkulturelle Güter. Ästhetische Produktionen als 
existenzbezogener Selbstausdruck werden dagegen aus dem objektivierten kulturellen Wissensreservoir ausgeklammert. Bedeutung erhalten die Kulturgüter durch ihre kontinuierliche Wiederholung und eine heteronome gesellschaftliche Wertsetzung. Die gesellschaftlich sinnstiftende Ordnungsbildung durch kulturelle Güter und Praxen im Horizont des segregierenden, sektoralen Kulturverständnisses wird durch eine Staffelung nach Schwierigkeits- und Anspruchsgraden gestützt. Klar abgegrenzte, feldspezifische Expertisen bilden den Strukturierungsmechanismus für die Differenzierung des sozialen Feldes.

Fokussiert werden reproduzierende Praxen im konventionellen und hochkulturellen Spektrum, denen die Funktion der Stiftung gesellschaftlicher Kohärenz bei gleichzeitig segregierender Ordnungsbildung zukommen. Die Verknüpfung mit einem Begabungsmodell naturalisiert und legitimiert die über diese Praxen hergestellte Ordnung. Dabei verbleiben die Praxen im Nahraum der eigenen sozialisatorischen Erfahrungen. Ihnen wird zwar Individualität, nicht aber Subjektivität, d.h. eine resubjektivierende Zuweisung von Bedeutungen unterstellt. Während der Einzelne rezeptiv an Kultur-Produktionen teilhat, darüber in Gemeinschaft eingebunden wird und als Mitglied einer Gemeinschaft seine gesellschaftliche Position erhält, sind diese Praxen des Einzelnen ohne Möglichkeit der Einwirkung auf Gemeinschaft und Gesellschaft entworfen. Kultur- bzw. Kunstproduktion ist vielmehr auf die Übernahme bestehender Normen und die richtige Anwendung von Techniken reduziert.

Ähnlich wie in Typ I, den Agenten des Verweilens im kulturellen Status Quo, liegt die Verantwortung für die Gestaltung gesellschaftlicher Ordnungsverhältnisse bei heteronomen Instanzen, insbesondere für das Handeln in professionellen Bezügen sind externe Legitimierungen maßgeblich. Dies zeigt sich beispielsweise daran, dass für den eigenen Erfahrungsraum der Universität und das Lernen in ihr eine Servicehaltung eingenommen wird, die die Lieferung von Unterrichtsgegenständen erwartet, die direkt in Schule zu übertragen sind. Es geht um eine Legitimierung von schulischem Vermittlungswissen durch universitäre Adelung.

In dieser Passage aus Biene (siehe Abb. 2) zeigt sich eine handlungsleitende Erwartung dahingehend, dass die Gegenstände im Studienfach Soziologie als Unterrichtsgegenstände in der Grundschule Verwendung finden können - die ganze Passage ist in einem ironischen Tonfall entwickelt, in welchem die Distanz zur Universität als geteilter Ort der Wissensproduktion erkennbar wird.

Für den Typ II ist ein positivistisches und quantitativ akzentuiertes Wissenskonstrukt grundlegend, hinsichtlich des Könnens eine Anerkennung gestaffelter Expertise. In didaktischer Hinsicht liegt damit der Akzent auf einem Entwurf von Lernen als Addition von Wissenselementen durch Anschauung und Beobachtung sowie als Übernahme hochkultureller Praktiken durch Üben. Kulturvermittlung ist als reproduzierende Praxis gefasst, die als Abarbeitung einer sachbezogenen Aufgabenstruktur und in der Anerkennung eines begrenzten Kanons entworfen ist. Sie läuft auf eine unveränderte Wiederholung des Vorfindlichen hinaus, denn sie wird als kontinuierliche Fortsetzung sozialisatorischer Vorprägungen verstanden. Lernen ist auf das nahräumliche Mit- und Nachmachen beschränkt, es ist eine Didaktik des mitgängigen Lernens und der Konstruktion ,richtigen Verstehens“ im Umgang mit kulturellen Objektivationen. Kulturelle Vermittlung ist Erziehung zur Traditionsbewahrung, eine Perpetuierung der eigenen Sozialisation ohne reflexive Distanz. Als 


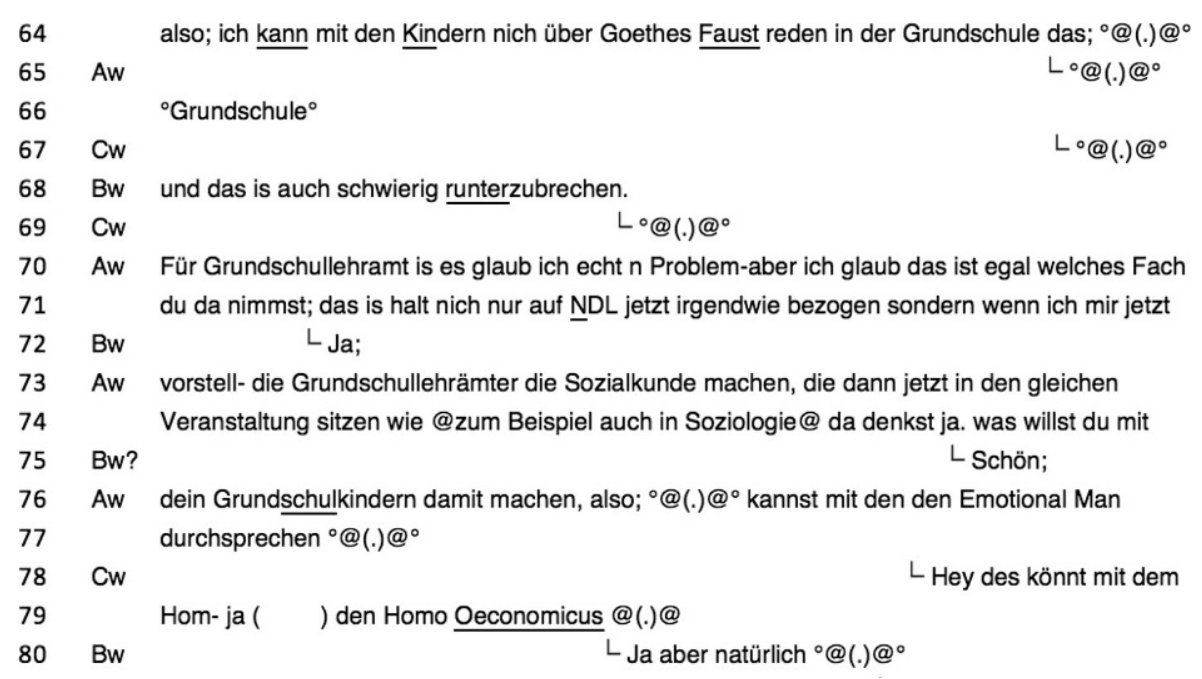

Abb. 2 Transkriptausschnitt Gruppe Biene

Effekt des Verbleibens in den nahräumlichen Erfahrungen wird kulturelles Professionshandeln zur Reproduktion exkludierender Praxisgemeinschaften.

\subsubsection{Typ III: Orientierung an Steigerung der Kunstexpertise in exklusiven Settings - Passanten kultureller Abgeschiedenheit}

Für den Typ III bilden die eigenen hochkulturellen Praxen den Ausgangspunkt und die Zielperspektive der Konstruktionen von Kultur. Es sind Praxen der invarianten Repetition. Sie sind an exklusiven konventionellen Kulturobjektivationen ausgerichtet, und als segregierendes Prinzip der sozialen Ordnungsbildung bestimmt. Dabei werden die Praxen auf das Selbst und seine sozialisatorische Einbettung fokussiert. Es resultiert eine Konzentration auf exklusive Praxisgemeinschaften. Wie für Typ II, den Agenten nahräumlicher Erfahrungen, ist ein sektorales und konventionelles Kulturverständnis grundlegend, mit dem kein Verständnis von kultureller Praxis als subjektivierende und subjektivierter Ausdruck des Selbst verbunden ist.

Die Konstruktionen einer Relationierung von Selbst mit Gemeinschaft ist dominant und gegenüber gesellschaftlichen Bezügen ausschließlich. Letztere erlangt letztlich keine Relevanz. Dies zeigt sich anhand des Entwurfs des kulturell aktiven Selbst im Rahmen sozialisatorischer Bahnen, der mit einer Indifferenz gegenüber unterschiedlichen Lernkontexten einhergeht und deren Unterschiede zum Verschwinden bringt. Das Studium und sein fachlicher Zuschnitt werden mit den familiären Selbstverständlichkeiten und Traditionen der hochkulturellen Praxis begründet, eine Professionsperspektive oder die Anerkennung institutioneller Besonderheiten der Universität dagegen negiert. Leitend ist die eigene Selbstverwirklichung, der die semantische Befolgung externer Normen untergeordnet wird.

Offensichtlich zeigt sich bei Käfer (siehe Abb. 3) in dieser Passage, dass das Studium mit seinen hohen praktischen Anteilen die ,perfekte Kombination“ (Z 89) 
83 Bw Ja ich find des auch voll; entspannt, oder; also für mich ist des super- son Studium mit nem

84 richtig großen praktischen Anteil, und dann aber noch das Theoretische, also- ich weiß nicht

$85 \mathrm{Cw} \quad$ L Mhh;

86 Bw ob ich; (1) $\underline{\text { soo }}$ studieren könnte; jetzt irgend $\mathrm{n}$ anderes Fach wiie; (.) Politikwissenschaften

87 oder so; das wär mir einfach viiel zu theoretisch; einfach sch-nur lernen; nur ständig dasitzen;

88 und- sich irgendwiie Informationen in sich rein@fressen sozusagen@ (.) ääh und das ist halt

89 mit Kunst irgendwie die perfekte Kombination, dass man (.) irgendwie so viel Praktisches

$90 \mathrm{CW}$

LJa;

Abb. 3 Transkriptausschnitt Gruppe Käfer

bietet, und theoretisches Lernen, das im negativen Gegenhorizont verhandelt wird, die Beibehaltung der Routinen nicht irritiert. Darauf ausgerichtet, im sozialisatorischen Nahraum exklusiver Kulturpraxis zu verbleiben, ist für Typ III kennzeichnend, dass zur Universität als Ort der Wissensproduktion kein Zugang erreicht wird. Es geht lediglich um eine fortgesetzte Anhäufung von Wissensbeständen in den vorfindlichen Spuren des habituell Gewordenseins. Modifizierte Erwartungshorizonte fallen unter das Verdikt der Ignoranz. Typ III verweigert eine professionelle Perspektive, ist dagegen ausschließlich daran orientiert, kulturelle Abgeschiedenheit in exkludierenden und elitären, also letztlich das Selbst limitierenden Verbänden aufrecht zu erhalten.

\subsubsection{Typ IV: Orientierung an transformativer Anregung in alternierenden Gemeinschaftsbildungen - Agenten hybridisierender Transformation}

Für Typ IV ist die Unabgeschlossenheit von Kultur für auf sie bezogene Konstruktionen konstitutiv. Konstruktivität und die Veränderlichkeit kultureller Ordnungsbildung wird grundsätzlich anerkannt. Dies zeigt sich daran, dass alle Dimensionen und denkbaren Bezugsfelder von Kultur als möglicherweise plural, mehrdeutig und wandelbar entworfen sind. Dieser pluralitätsorientierte Zugang ist mit einer Anerkennung divers fundierter Werthaltungen und ihrer Wandelbarkeit verbunden. Solchermaßen wird Alterität in Rechnung gestellt, die aber in einem Netz variabler Differenzen aufeinander bezogen bleibt. Dieses ausdifferenzierte und dynamische Netz von Bedeutungen ist nicht hierarchisch angelegt, dadurch, dass kulturelle Interpretationen und Ausdrucksformen aber an die Subjektivität des Einzelnen gebunden sind, bergen sie durch die Vielfalt relativer Differenzsetzungen durchaus auch Konfliktpotential. Kultur ist gerade nicht ein harmonistisches Feld, sondern die Arena der Austragung von Deutungsdivergenzen. Kultur ist ein temporäre Gemeinschaften stiftender Raum für diversifizierende Erfahrungen von Individuen in der Welt.

Kulturelle Produktion und Reproduktion sind als Veränderungsprozesse entworfen, als kontextualisierte Variationen. Es gilt, über kulturelle Praxen Anschlüsse des Einzelnen an gesellschaftliche Netzwerke mit Gemeinschaftsanteilen in je unterschiedlichem Situations- und Gegenstandsbezug diskursiv und mit temporärer Gültigkeit zu erarbeiten. Für Typ IV ist der Fortbestand von Traditionen durchaus von Bedeutung, er ist aber an die je individuellen, adaptierten und modifizierenden Realisierungen gebunden, kurz, an re-subjektivierende Praxen: Tradition ist Veränderung, nicht Stillstand. 
Das Selbst ist als Handelndes in seinen gemeinschaftlichen Relationen, und dadurch als gesellschaftlich Gestaltendes entworfen. Als Gestaltendes steht das Selbst in der Verantwortung für das Überschreiten des eigenen Erfahrungshorizontes, bspw. durch Lernen. In gesellschaftlicher Hinsicht ist mit der Perspektive auf Verantwortung ein grundlegend partizipatorischer Zugang zu Institutionen verbunden, der auf die Partizipation aller Beteiligten ausgedehnt wird und zugleich deren partizipativen Rechte anerkennt. Vor dem Hintergrund einer mehrdimensionalen Konstruktion professionellen Handelns wird die Ausführung der Profession als institutionelle Rolle mit individuellen Handlungsspielräumen entworfen und der handelnden Person eine umfassende Verantwortung für vielfältige Lern- und Bildungsprozesse der Schülerinnen und Schüler zugerechnet.

Epistemisch ist Wissen grundlegend als von Mehrdeutigkeit und Pluralität strukturiert entworfen. In diesem Entwurf werden verschiedene Wissensebenen unterschieden, bleiben aber aufeinander bezogen. Wissen hat in verschiedenen Dimensionen, als theoretisches, als handlungsreflexives, als Erfahrungswissen Relevanz, es ist kein abgeschlossener Bestand und geht mit Nicht-Wissen einher. Mit dieser epistemologischen Grundlegung ist kulturbezogenes Lernen als Bildung entworfen, die in zweierlei Hinsicht bedeutsam ist: Als produktiver Umgang mit Traditionen durch eine sie wandelnde Aneignung, und als Steigerung der eigenen Komplexität durch die Verflechtung praktischer Erfahrungen mit Selbst- und theoretischer Reflexion. Beide Aspekte sind miteinander verschränkt, denn die vorgängige Enkulturation ist als eine der Reflexion zugängliche und damit bearbeitbare entworfen.

Tradierung und Komplexitätssteigerung fallen ineinander, wie bei dieser Passage der Gruppe Skorpion in diesen wenigen Zeilen erkennbar wird (siehe Abb. 4), denn die Konstruktivität und Pluralität von Kultur ist für sie selbstverständlich Unterrichtsgegenstand wie -prinzip, und Scheu, eine neue Kultur zu entwickeln (Z 160), zeigt sich nicht. Lernen wird transformatorische Wirkung zugeschrieben, die gleichwohl in ihrem Ergebnis nicht bestimmbar ist: Vielmehr ist mit einem offenen Ausgang zu rechnen. Für den Typ IV ist kulturelles Professionshandeln mit der Aufgabe verbunden, Kultur in ihrer möglichen Bedeutungsbreite relevant werden zu lassen. Sie ist Reflexionsanlass für die Aneignung von Orientierungswissen.

Professionalität im Kontext kultureller Bildung stützt für sich bei Typ IV auf die Orientierung an transformativen Anregungen, die zur Steigerung von sozialer Hybridität in alternierenden Gemeinschaftsbildungen beitragen: Es sind Agenten hybridisierender Transformation.

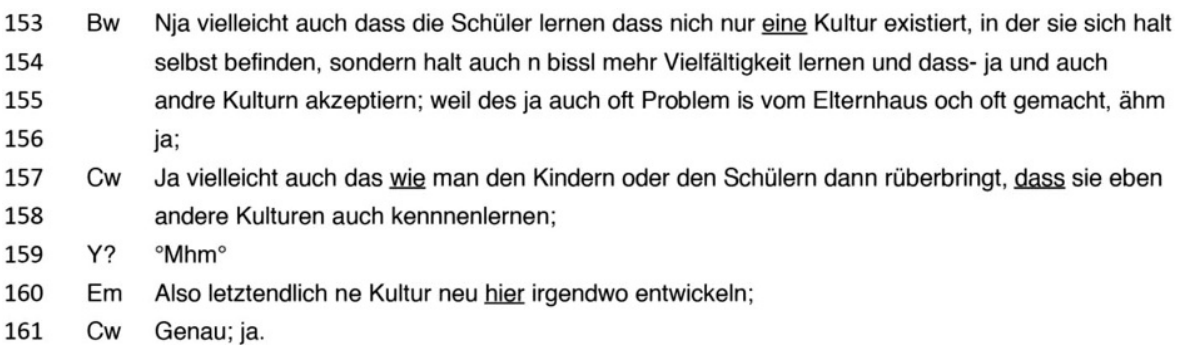

Abb. 4 Transkriptausschnitt Gruppe Skorpion 


\subsubsection{Typ V: Orientierung an beiläufigem Arrangieren als Kontingenzbewältigung für Kollektivsubjekte - Passanten ratloser Problematisierung}

Für die Konstruktionen von Welt im kulturellen Feld von Sinn und Bedeutung sind für diesen Typ zwei Aspekte grundlegend: Zum einen ist Kultur als allumfassender Differenzmarker ethno-kultureller Zugehörigkeit leitend, als solcher wird Kultur sozialisatorisch, vor allem im Kontext der Familie, erworben. Sie erstreckt sich auf den Bedeutungs- und Normgehalt gemeinschaftlicher Gewohnheiten und Gebräuche. Kennzeichen wie Kleidung aber auch Bedeutungssysteme wie Religion charakterisieren die jeweilige Kultur als durchgängig unterschiedliche zu anderen. In dieser Hinsicht gleicht dieser Orientierungsgehalt denen des Typ I, den Agenten des Verweilens im Status Quo. Der zweite Orientierungsaspekt für Typ V scheint dem gegenüber geradezu gegenläufig zu sein, nämlich die systematische Unterscheidung von kultureller Gemeinschaft und kulturell pluraler Gesellschaft in den Konstruktionen der Sozialordnung. Die Zugehörigkeit zu einer Gemeinschaft eines intern homogenen Kollektivsubjektes bietet Beheimatung für den Einzelnen, während Gesellschaft anerkanntermaßen von der Anwesenheit mehrerer, als prinzipiell unterschiedlich gekennzeichneter Kollektivsubjekte gekennzeichnet ist. Aus dieser systematischen Trennung von Gemeinschaft und Gesellschaft resultiert eine Irritation der Gewissheit über die eigene Zugehörigkeit, die in fortwährende Klärungsversuche mündet, was Kultur sei.

Für den Typ sind die kulturbezogenen Praxen der Produktion und Reproduktion auf die Begegnung mit kanonisch entworfenen kulturellen Objektivationen fokussiert und von invarianter Rezeption konturiert. Tragend ist ein Verständnis kultureller Produktion als erhaltender Reproduktion routinierter Handlungen, die in der Sozialisation eingeübt werden. Dies führt zwangsläufig zur unveränderlichen Repetition eines tradierten Bestandes kultureller Objektivationen und immaterieller Kulturgüter, wie sie sich auch im Typ II, den Agenten nahräumlicher Erfahrungen zeigt. Kulturelle Praxis schafft segregierende Distinktionsmerkmale, die eine ordnungsstiftende Funktion haben. Daneben wird kulturelle Praxis hier aber auch als Selbstausdruck anerkannt: Diese Konzeptionierung eröffnet Spielräume für Modifikationen und Variationen. Die Pluralität der Ausdrucksweisen wird allerdings als Irritation der Wohlordnung des Ganzen erfahren.

Angesichts der systematischen Unterscheidung von Gemeinschaft und Gesellschaft und der hohen Bedeutung, die gemeinschaftsfundierter Zugehörigkeit beigemessen wird, wird Kultur als verpflichtendes Lernfeld expliziert. Die Zugehörigkeit des Selbst ist nicht nur sozialisatorisch vorgespurt, sie trägt auch wandelbare Züge, und muss immer wieder neu hergestellt werden. Es zeigt sich, dass die eigene Zugehörigkeit aufgrund der Anwesenheit kulturell Anderer als prekär wahrgenommen wird. Kultur steht für Irritationen, sie ist ein Unruheherd. Hieraus erwächst angesichts einer Selbstpositionierung als verantwortliche Pädagogen und Pädagoginnen ein Handlungsimperativ, der damit konfrontiert ist, dass für den professionellen Kontext eine Defizitperspektive angesichts des eigenen Erfahrungs- und Wissenshaushalt eingenommen wird. Nicht-Können und Nicht-Geliefert bekommen bilden den Fokus der professionsbezogenen Selbst-Welt-Relationierung. 
Bw Und es is halt auch total schwierig, weil [räuspert sich] wenn ich jetzt so überlege; also ich hab einmal die Kinder die da total aufgeschlossen sind; aber wenn ich mir jetzt meine Eltern ankucke, is es gaanz oft so, wenn ich in irgendnem Stadtteil unterwegs bin oder dann ja- dann is einfach total schwierig wenn da so (.) ganz viele ähm in ner anderen Sprache sprechen oder so dann ham die total grad wenns dunkel is oder so halt sofort so Ängste; und des stell ich bei mir selber so nich fest, und auch wenn ich mit Freunden unterwegs bin abends auch überhaupt nich, aber die ältere Generation hat da halt wirklich nochn Problem mit; und; ich glaub das is

Dw L ${ }^{\circ M h m ; \circ}$

Bw eben auch so (.) sowas wo ich so denke naja, da is einfach schwierig; weil die Kinder werden damit jetzt extrem konfrontiert; wir werden damit extrem konfrontiert; weil wir einfach viel in der Uni auch damit zu tun haben; oder uns eben- ja mit uns selbst und der Lehrerbildung beschäftigen, aber die ältere Generation davon wirklich zu überzeugen dass es auchn positiven Effekt haben kann auf unsre Gesellschaft, is halt wirklich schwierig. und ähm ja grad wenn man so auch an die Großeltern denkt dass sich so einiges verändert; und dass wir halt multikulturell geworden sind; da irgendwo oder. (.) ja; also ich glaub das is- da ham wir halt irgendwie noch

Abb. 5 Transkriptausschnitt Gruppe Maikäfer

Im nachfolgenden Transkriptausschnitt aus der Gruppe Maikäfer wird deutlich, dass sich die Gruppe letztlich als Angehörige der älteren Generation, ja gar ihrer Großeltern verortet, und die zu überzeugen, ,dass sich so einiges verändert; und dass wir halt multikulturell geworden sind“ (Z 121 f) ,,is halt wirklich schwierig“ (Z 120). Es bleibt eine Ratlosigkeit, wie im Generationenwechsel die theoretische Anerkennung einer pluralisierten Gesellschaft in die Erfahrungshaushalte der Einzelnen eingehen kann (siehe Abb. 5).

Angesichts der hier sichtbar werdenden Konfliktlage zwischen einer kommunikativ-theoretischen Wertschätzung von Pluralität und Bedeutungsvielfalt und der gleichzeitigen Orientierung an der eigenen Zugehörigkeit zu einem homogenen Kollektivsubjekt resultiert für diesen Typ eine didaktische Strategie, die auf das Implizite, auf „kleine Lösungen“, zusammengefasst auf eine Didaktik der Zaghaftigkeit setzt. Auch wenn Didaktik durchaus als Veränderungshebel markiert ist, und Arrangements kultureller Vermittlung als Ansatzpunkte der sukzessiven Veränderung und Kontingenzbewältigung favorisiert werden, bleibt der Typ selbst davon gekennzeichnet, Problematisierungen ratlos ausgeliefert zu sein.

\section{Varianz und Kontingenz kultureller Deutungen - eine Diskussion der Ergebnisse und Konsequenzen für die Lehramtsbildung}

Dynamiken im kulturellen Netz der Bedeutungen stellen das grundlegende Problem in den Gruppendiskussionen, und damit die zentrale Vergleichsachse dar. Die Gruppen arbeiten sich daran ab, sich zu Varianz und Kontingenz kultureller Deutungen in ein für sie stimmiges Verhältnis zu setzen. Wie dargestellt, wurde diese Auseinandersetzung anhand verschiedener Themen, vor allem aber in unterschiedlicher Weise realisiert. Dabei zeigen die fünf Orientierungstypen teils Gemeinsamkeiten, teils Kontraste: Je unterschieden sind sie in den Kombinationen verschiedener Orientierungsaspekte. Im Blick auf das Forschungsinteresse für die Orientierungen von 
Lehramtsstudierenden, die für ihr professionelles Handeln in seiner kulturellen Dimensionierung relevant sind, sollen die Typen zunächst vergleichend hinsichtlich ihrer Kulturkonstruktionen und den Effekten, die diese Konstruktionen für professionelles Handeln haben können, rekapituliert werden. Im Anschluss wird das zentrale Ergebnis, dass die dominanten Deutungen der Mehrheitskultur zumeist in unreflektierten Wiederholungen zur Geltung kommen, zum Anlass genommen, erste Überlegungen zu Konsequenzen für die Lehramtsausbildung zu formulieren. Vorgeschlagen wird, kulturelle Reflexivität als Professionalisierungsperspektive bereits im Lehramtsstudium zu verankern.

\subsection{Essenzialisierende und konventionelle Konstruktionen von Kultur}

Den Typen I und V ist eine Fokussierung auf einen essentialisierenden Entwurf von homogenen Kulturkonstruktionen, mit einer Bestimmung von Alterität als ethno-kultureller, eigen. Dieses Kulturverständnis mündet in zwei unterschiedliche Entwürfe professionellen Handelns: Zum einen wird kulturelle Tradierung als unveränderliche Wiederholung und instruktive Einpassung in vorgängige Ordnungen entwickelt (Typ I). Für den anderen Orientierungstyp erweist sich dies angesichts der faktischen Pluralisierung kultureller Bedeutungssysteme und Zugehörigkeitsoptionen als problembehaftet (Typ V). Diese Orientierung ist mit einem Verständnis von Didaktik als „Didaktik der Zaghaftigkeit“" verbunden. Studierende mit dieser Orientierung können kaum konstruktive didaktische Strategien entwickeln, auch wenn sie im theoretisch-kommunikativen Wissen deren Notwendigkeit anerkennen.

Einen weiteren Schwerpunkt bilden Orientierungen, die hinsichtlich ihrer Kulturkonstruktionen von einem konventionellen Kulturkonstrukt ausgehen, und zwar in einer Fokussierung auf hochkulturelle Kulturobjektivationen (Typ II und III). Diese Orientierung führt zu einer Konzentration auf die Kontinuität der eigenen, vornehmlich kulturreproduzierenden Praxis. Die Ausübung von Kultur wird als eine exklusive, andere ausschließende und nach innen Gemeinschaft schaffende Praxis gedacht, Vermittlungsambitionen werden auf die Gleichen und schon Dazugehörigen konzentriert (Typ III).

Typ IV steht für Konstruktionen, in die die Konstruktivität von Kultur, gesellschaftliche Hybridformationen und dynamische Relationierungen einfließen. Kultur ist als veränderbare und nicht-statische gerahmt. Es zeigt sich eine Anerkennung von Mehrfachzugehörigkeit, von Partizipationsambitionen und -rechten aller sowie deren Verbindung zur Anerkennung von Entäußerungsvarianten des Individuums in kulturellen Produkten. Eine kulturell vielfältige, inkludierend und partizipative professionelle Praxis schließt sich an.

Im Hinblick auf Alterität zeigen sich für Typ I, II und III Parallelen. Der Umgang hiermit ist problembehaftet, insofern sie als Abweichung das statisch entworfene Ordnungssystem irritiert. Während Typ III Alterität umfassend ignoriert, arbeiten Typ I und II an ihrer Minimierung bzw. Ausgrenzung. Typ V besetzt in dieser Hinsicht eine besondere Stelle: Ausgehend von dem auf Gemeinschaft bezogenen Konstrukt intern homogener Kollektivsubjekte werden diese im Blick auf Gesellschaft pluralisiert. Es ist ein Nebeneinander verschiedener Kollektive. Angesichts der faktischen Pluralisierung kultureller Bedeutungssysteme und Zugehörigkeitsop- 
tionen wird kulturelle Tradierung als unveränderliche Wiederholung, wie sie für Typ I und II handlungsleitend ist, dann aber problembehaftet. Zwar wird kulturelle Vermittlung als Veränderungshebel in der transgenerationalen Weitergabe expliziert, dass sie aber im handlungsleitenden Wissen nicht verankert ist, zeigt sich an der Ratlosigkeit in Bezug auf professionelle Handlungsoptionen.

\subsection{Verhaftet im Herkunftsmilieu - Reproduktionswege mehrheitskultureller Dominanz}

In der Rückbesinnung auf das gemeinsame Moment aller Gruppen, ihre Positionierung angesichts von Varianz und Kontingenz kultureller Deutungen und den aus ihnen resultierenden Veränderungsimpulsen muss resümiert werden, dass von den fünf Typen einer dynamische Momente in kultureller Hinsicht ignoriert (Typ III), zwei weitere versuchen, ihnen mit einem Insistieren auf Vorfindliches zu begegnen (Typ I und II), ein Typ sich als ratlos zeigt (Typ V) und lediglich einer Modifikationen, Anpassungen und Veränderungen als genuin kulturelle Phänomene entwirft (Typ IV). ${ }^{3}$ Dieser Typ ist daran orientiert, Veränderungsimpulse aufzugreifen und in professioneller Hinsicht sich selber als tätiger Akteur für die Produktion von Veränderungen zu entwerfen. Vier der fünf Typen sind dagegen daran orientiert, die ihnen vertrauten kulturellen Bedeutungszusammenhänge in jeder Hinsicht zu erhalten und die nachwachsende Generation in genau diese einzuführen. Sie sind ihrem Herkunftsmilieu verhaftet, ohne Ansätze einer reflexiven Distanz oder der Verhältnissetzung zu ihrer eigenen Biographie, zu ihrem kulturellen Gewordensein. In verschiedenen Varianten entwerfen sie eine exkludierende Didaktik, eine des Bewahrens und Einpassens oder auch eine der ratlosen Zaghaftigkeit für das professionelle Handlungsfeld. Die empirisch gestützten Orientierungsmomente verweisen vielmehr darauf, dass sie keine Distanz schaffende Perspektive auf ihre Tätigkeit als Doing Culture by Being a Teacher einnehmen. Angesichts der Kennzeichnung von Schule als Ort der Enkulturation und der Anforderung an Schülerinnen und Schüler, sich im Prozess der Personwerdung zwischen multiplen Ordnungen und einer Vielzahl von Sinnofferten der Gesellschaft erst zuzuordnen, aber auch angesichts der paradoxen Struktur, dass Schule in Kulturen einführt, dies aber nur über eine Kultur realisieren kann, offenbart sich in diesem Ergebnis eine problematische Diskrepanz: Für vier der fünf rekonstruierten Orientierungstypen äußern sich die Positionierungen gegenüber kulturellen Dynamiken als die einer großen Distanz und Fremdheit. Vielmehr verhelfen sie durch den Modus der Bewahrung der Mehrheitskultur ihres Herkunftsmilieus ungebrochen zur Geltung, was zunächst machttheoretisch zu kritisieren wäre, aber auch perspektivisch dahingehend ein Problem darstellt, dass die gesellschaftlich integrierende Funktion der Schule als Segregation realisiert wird, und Schule dadurch als Reproduktionsagent soziale Ungleichheit verstärkt (vgl. Kramer und Helsper 2010).

\footnotetext{
3 Die Ausdifferenzierung von fünf Typen, von denen vier an der Abwendung von Varianz orientiert sind, ergibt sich aus dem vorliegenden Material. Es ist nicht auszuschließen, dass mit weiteren Erhebungen auch die Akzeptanz und Beförderung von Varianz, wie sie sich hier nur in einem Typ (IV) zeigt, weiter auszudifferenzieren ist.
} 


\subsection{Kulturelle Reflexivität als Professionalisierungsperspektive}

Die Orientierungen der angehenden Lehrkräfte für das kulturelle Professionshandeln resultieren als Teil ihrer impliziten Wissensbestände aus Handlungsroutinen in geteilten Erfahrungsräumen. Als strukturierende Struktur sind sie biographisch aufgeschichtet, und nicht kurzfristig abzulegen, noch könnten sie durch intentionale Vermittlungsambitionen im Rahmen des Studiums ,umgesteuert“ werden. Spielräume eröffnen sich gleichwohl dort, wo durch eine Veränderung des Erfahrungsraumes die bisherigen Handlungsroutinen Irritationen erfahren und auch habituelle Elemente einem Anpassungsdruck unterliegen, ist doch davon auszugehen, dass bereits institutionelle Wechsel zu Transformationen führen können, z. B. aus einem Schüler- ein Berufs- und schließlich ein Professionshabitus resultiert (vgl. Helsper 2018). Dass derlei Irritationen nicht auf ein bestimmtes Fächerspektrum oder spezifische Studienanteile zu beschränken wären, betonen Costa et al. in ihrem Resümee, in welchem insbesondere der Anteil der Fachdidaktiken hervorgehoben wird (Costa et al. 2018).

Reflexivität als Kennzeichen von Professionalität wird im Kontext der Professionsforschung vielfältig thematisiert. Zumeist bezieht sie sich auf Kompetenzen des theoretischen Nachdenkens über die eigene Unterrichtspraxis, die in der ersten Phase der Lehramtsausbildung angebahnt werden sollten. Teils geht es um Reflexivität als „,nachträgliche[r] Deutung“, bezogen auf das unterrichtliche Handeln (Combe und Kolbe 2004, S. 865), oder auch um ein ,systematisiertes Reflexionswissen, das die eigenen Entscheidungen zu begründen und zu legitimieren, zu kontrollieren und gegebenenfalls zu korrigieren vermag." (Radtke 2000, S. 2). Während Combe und Kolbe von einem kollektiv erworbenen Praxiswissen sprechen, betont Radtke den universitären Erwerb von Wissenskomponenten. Gleichwohl hat sich auch in der hier vorgestellten Studie gezeigt, dass die Theorie-Praxis-Spannung im akademischen Lernen seitens der Studierenden bislang kaum auf transformative Bildungsprozesse in der Perspektive auf einen Berufshabitus gerichtet wird. Deswegen bedarf es weitergehender Überlegungen zu Formen und Inhalten der akademischen Lehre, damit sie für die Studierenden bedeutsam werden kann. Lehre, die die biographischen Erfahrungen wie die gegenwärtigen praktischen Erfahrungen erkennbarer einbezieht, scheint für kulturelles Professionshandeln weiterführend (von Rosenberg 2016). Dies umfasst mehr als der inhaltlich orientierte Vorschlag Klepackis, ,,auch [die] universitäre Lehrerbildung selber als Kulturelle Bildung [zu] denken“ (Klepacki 2014, S. 4). Um biographisch-habituell relevant zu werden, müssten Erfahrungsräume geschaffen werden, die als praktische die habituellen Routinen irritieren, und an vielfältige Formen des Wissens und des Wissens über Wissensformen im Sinne einer MetaReflexivität explizit angeschlossen werden (vgl. Cramer et al. 2019). Dabei geht es nicht nur um eine erfahrungsbasierte Verankerung in den Wissensbeständen der Studierenden, sondern vielmehr auch darum, den eigenen kulturell-habituellen Horizont zum Gegenstand der Reflexion zu transzendieren, um seine Durchschlagkraft reflexiv eingrenzen zu können (vgl. Hericks et al. 2018; Timm und Scheunpflug 2020). Die Notwendigkeit einer solchen Reflexivität, die als ein Amalgam theoretischer Annäherungen, sinnlicher und emotionaler Erfahrungen - als ein auch intuitives Wissen der eigenen Begrenztheit und des eigenen Irritationsvermögens - verstanden werden kann, speist sich auch daraus, dass Lehrkräfte angesichts der glokalen Situ- 
iertheit ihres Handelns die eigene Komplexität erhöhen müssen (vgl. Lang-Wojtasik 2014; Scheunpflug 2016). Vorgeschlagen wird deswegen, das Modell des ,reflective practioner" (Schön 1987) dahingehend zu erweitern, dass Lehramtsstudierende im Rahmen ihrer akademischen Ausbildung zu Erfahrungsbereichen durchdringen, die sie dazu herausfordern, den kulturellen Horizont des eigenen Werdens zu überschreiten.

\subsection{Ausblick}

Um Limitationen, die sich aus der Samplestrukturierung, der methodologischen Abstrahierung von Biographien und der regionalen Begrenzung ergeben, zu begegnen, bietet es sich an, für eine weitergehende Fundierung genannter Impulse für die Lehrerbildung, über Studien nachzudenken, in denen vor allem die Entstehungskontexte, möglicherweise auch die Verteilung der aufgezeigten Orientierungstypen nachgezeichnet werden können. Ergiebig könnte zudem die Erweiterung eines Samples um Gruppen mit ausgeprägten Befremdungs- oder Irritationserfahrungen oder solchen mit einer Vermittlungsperspektive jenseits der institutionell geformten Lehramtes sein, ebenso Forschungsperspektiven, in denen biographische Erfahrungs- und Transformationsfelder erschlossen werden könn(t)en. Thematische Anschlüsse zeigen sich angesichts der Schnittstellen des Umgangs mit Varianz und Kontingenz auch für Globales Lernen und in diesem Kontext erhobener Orientierungen von Lehrkräften. Aus zwei Gründen ist zudem eine präzisere Bezugnahme auf internationale Forschungszusammenhänge ratsam: Als national geprägte steht die Lehrkräftebildung zum einen vor der Herausforderung, auf ein national formiertes Schulsystem in einer globalisierten Welt vorzubereiten, und damit in kulturell-historischer Gebundenheit genau diese zu überschreiten. Zum anderen könnten in grenzüberschreitenden Vergleichen kontextspezifische wie kontextübergreifende Tendenzen voneinander geschieden werden, um sie einer am Kontext orientierten Bearbeitung wie einer generellen zugänglich zu machen.

Danksagung Ich danke den anonymen Gutachtenden für die zahlreichenden Hinweise, die zu einer deutlichen Weiterentwicklung des Textes geführt haben.

Funding Open Access funding enabled and organized by Projekt DEAL.

Open Access Dieser Artikel wird unter der Creative Commons Namensnennung 4.0 International Lizenz veröffentlicht, welche die Nutzung, Vervielfältigung, Bearbeitung, Verbreitung und Wiedergabe in jeglichem Medium und Format erlaubt, sofern Sie den/die ursprünglichen Autor(en) und die Quelle ordnungsgemäß nennen, einen Link zur Creative Commons Lizenz beifügen und angeben, ob Änderungen vorgenommen wurden.

Die in diesem Artikel enthaltenen Bilder und sonstiges Drittmaterial unterliegen ebenfalls der genannten Creative Commons Lizenz, sofern sich aus der Abbildungslegende nichts anderes ergibt. Sofern das betreffende Material nicht unter der genannten Creative Commons Lizenz steht und die betreffende Handlung nicht nach gesetzlichen Vorschriften erlaubt ist, ist für die oben aufgeführten Weiterverwendungen des Materials die Einwilligung des jeweiligen Rechteinhabers einzuholen.

Weitere Details zur Lizenz entnehmen Sie bitte der Lizenzinformation auf http://creativecommons.org/ licenses/by/4.0/deed.de. 


\section{Literatur}

Bauer, B., Beuter, K., Lindner, K., Hlukhovych, A., \& Vogt, S. (2018). Kulturbezogene Lehrerinnen- und Lehrerbildung. Grundlegungen, Perspektiven und Operationalisierungsoptionen. In A. Hlukhovych, B. Bauer, K. Beuter, K. Lindner \& S. Vogt (Hrsg.), Kultur und kulturelle Bildung. Interdisziplinäre Verortungen - Lehrerinnen- und Lehrerbildung - Perspektiven für die Schule (S. 13-36). Bamberg: University of Bamberg Press.

Bockhorst, H., Reinwand-Weiss, V.-I., \& Zacharias, W. (Hrsg.). (2012). Handbuch Kulturelle Bildung. München: kopaed.

Bohnsack, R. (1999). Rekonstruktive Sozialforschung. Einführung in Methodologie und Praxis qualitativer Forschung. Opladen: Leske + Budrich.

Bohnsack, R. (2005). Standards nicht-standardisierter Forschung in den Erziehungs- und Sozialwissenschaften. In I. Gogolin, H.-H. Krüger, D. Lenzen \& T. Rauschenbach (Hrsg.), Standards und Standardisierungen in der Erziehungswissenschaft (Zeitschrift für Erziehungswissenschaft: Sonderheft 4, S. 63-81). Wiesbaden: Springer VS.

Bohnsack, R. (2006). Dokumentarische Methode. In R. Bohnsack, W. Marotzki \& M. Meuser (Hrsg.), Hauptbegriffe Qualitativer Sozialforschung (S. 40-44). Opladen: Barbara Budrich.

Bohnsack, R. (2013). Dokumentarische Methode und die Logik der Praxis. In A. Lenger, C. Schneickert \& F. Schumacher (Hrsg.), Pierre Bourdieus Konzeption des Habitus. Grundlagen, Zugänge, Forschungsperspektiven (S. 175-200). Wiesbaden: Springer VS.

Bohnsack, R., Przyborski, A., \& Schäffer, B. (Hrsg.). (2010). Das Gruppendiskussionsverfahren in der Forschungspraxis. Opladen: Barbara Budrich.

Combe, A., \& Kolbe, F.-U. (2004). Lehrerprofessionalität: Wissen. Können. Handeln. In W. Helsper \& J. Böhme (Hrsg.), Handbuch der Schulforschung (S. 833-851). Opladen: VS.

Costa, J., Kühn, C., Timm, S., \& Franken, L. (2018). Kulturelle Lehrerbildung - Professionalität im Horizont der Globalisierung. ZEP Zeitschrift für internationale Bildungsforschung und Entwicklungspädagogik, 41(4), 20-29.

Cramer, C., Harant, M., Merk, S., Drahmann, M., \& Emmerich, M. (2019). Meta-Reflexivität und Professionalität im Lehrerinnen- und Lehrerberuf. Zeitschrift für Pädagogik, 65(3), 401-423.

Dwyer, R. (2016). Music teachers' values and beliefs. New York: Routledge.

Fend, H. (2006). Neue Theorie der Schule. Einführung in das Verstehen von Bildungssystemen. Wiesbaden: VS.

Fink, T., Hill, B., Reinwand-Weiss, V.-I., \& Wenzlik, A. (Hrsg.). (2012). Die Kunst über Kulturelle Bildung zu forschen. München: kopaed.

Fuchs, M., \& Braun, T. (Hrsg.). (2016). Zur Ästhetischen Dimension von Schule. Die Kulturschule und kulturelle Schulentwicklung. Grundlagen, Analysen, Kritik (Bd. 2). Weinheim: Beltz Juventa.

Geertz, C. (2016). The interpretation of cultures. Selected essays (EPub edition). New York: BasicBooks.

Glaser, B. G., \& Strauss, A. L. (1967). The discovery of grounded theory: strategies for qualitative research. London: Weidenfeld and Nicolson.

Göhlich, M. (2007). Schulkultur. In H. J. Apel \& W. Sacher (Hrsg.), Studienbuch Schulpädagogik (3. Aufl., S. 104-120). Bad Heilbrunn: Julius Klinkhardt.

Hall, S. (1973). Encoding decoding in the television discourse (Discussion Paper). Birmingham: University of Birmingham.

Helsper, W. (2018). Lehrerhabitus: Lehrer zwischen Herkunft, Milieu und Profession. In A. Paseka, M. Keller-Schneider \& A. Combe (Hrsg.), Ungewissheit als Herausforderung für pädagogisches Handeln (S. 105-140). Wiesbaden: Springer VS.

Helsper, W. (2019). Vom Schüler- zum Lehrerhabitus - Reproduktions- und Transformationspfade. In R.-T. Kramer \& H. Pallesen (Hrsg.), Lehrerhabitus. Theoretische und empirische Beiträge zu einer Praxeologie des Lehrerberufs (S. 49-72). Bad Heilbrunn: Klinkhardt.

Hericks, U., Sotzek, J., Rauschenberg, A., Wittek, D., \& Keller-Schneider, M. (2018). Habitus und Normen im Berufseinstieg von Lehrer*innen - eine mehrdimensionale Typenbildung aus der Perspektive der Dokumentarischen Methode. ZISU, 7, 65-80.

Hirschauer, S. (2014). Un/doing Differences. Die Kontingenz sozialer Zugehörigkeiten. Zeitschrift für Soziologie, 43(3), 170-191.

Klepacki, L. (2014). Lehrerbildung als Kulturelle Bildung - ein kulturtheoretisch-geisteswissenschaftlicher Essay über Schule. In Kulturelle Bildung Online. https:/www.kubi-online.de/index.php/artikel/ lehrerbildung-kulturelle-bildung-kulturtheoretisch-geisteswissenschaftlicher-essay-ueber. Zugegriffen: 13. Juni 2017. 
Koerrenz, R. (2014). Bildung als Reflexion und Gestaltung von Vorurteilen. Globale Bildung und die Welt im Kopf. In A. Blichmann \& R. Koerrenz (Hrsg.), Pädagogische Reform im Horizont der Globalisierung (S. 13-28). Paderborn: Schöningh.

Kramer, R. T. (2015). „Reproduktionsagenten“ oder „Transformationsakteure“? Lehrkräfte im Blick der Bildungssoziologie von Pierre Bourdieu. Zeitschrift für Soziologie der Erziehung und Sozialisation, 35(4), 344-359.

Kramer, R.-T., \& Helsper, W. (2010). Kulturelle Passung und Bildungsungleichheit. Potenziale einer an Bourdieu orientierte Analyse der Bildungsungleichheit. In H.-H. Krüger (Hrsg.), Bildungsungleichheit revisited (S. 103-125). Wiesbaden: VS.

Kramer, R.-T., \& Pallesen, H. (Hrsg.). (2019). Lehrerhabitus. Theoretische und empirische Beiträge zu einer Praxeologie des Lehrerberufs. Bad Heilbrunn: Klinkhardt.

Krenz-Dewe, D. (2017). Kultur und Bildung - eine herrschaftskritische Betrachtung mit den Cultural Studies. In G. Weiß (Hrsg.), Kulturelle Bildung - Bildende Kultur. Schnittmengen von Architektur und Kunst (S. 415-426). Bielefeld: transcript.

Lanfranchi, A. (2008). Interkulturelle Kompetenz als Element pädagogischer Professionalität - Schlussfolgerungen für die Lehrerausbildung. In G. Auernheimer (Hrsg.), Interkulturelle Kompetenz und pädagogische Professionalität (2. Aufl., S. 231-260). Wiesbaden: VS.

Lang-Wojtasik, G. (2014). Global teacher für die Weltgesellschaft!? Theoretische Überlegungen zu den Kompetenzen von Lehrkräften, um Globales Lernen kompetenzorientiert zu unterrichten. ZEP Zeitschrift Für internationale Bildungsforschung und Entwicklungspädagogik, 37(3), 4-9.

Lehtomäki, E., Janhonen-Abruquah, H., \& Kahangwa, G. L. (Hrsg.). (2017). Culturally responsive education. Reflections from the global south and north. London: Routledge.

Liebau, E. (2013). Forschung zur Kulturellen Bildung in Deutschland: Bestand und Perspektiven (Projektbericht). Erlangen: Friedrich-Alexander-Universität Erlangen-Nürnberg.

Liebau, E. (2019). Kulturelle Bildung. Die Schule als genuinen Kulturort denken. Schulmanagement, 5, $8-11$.

Loos, P., \& Schäffer, B. (2001). Das Gruppendiskussionsverfahren. Opladen: Leske + Budrich.

Mecheril, P. (2008). Kompetenzlosigkeitskompetenz‘. Pädagogisches Handeln unter Einwanderungsbedingungen. In G. Auernheimer (Hrsg.), Interkulturelle Kompetenz und pädagogische Professionalität (2. Aufl., S. 15-34). Wiesbaden: VS.

Meister, N. (2018). „Die sollen Spaß dran haben!“ Professionsverständnis und kollektive Orientierungen von Lehramtsstudierenden des Faches Sport. In T. Leonhard, J. Košinár \& C. Reintjes (Hrsg.), Praktiken und Orientierungen in der Lehrerbildung. Potentiale und Grenzen der Professionalisierung (S. 224-238). Bad Heilbrunn: Julius Klinkhardt.

Prange, K. (1978). Pädagogik als Erfahrungsprozess. Teil 1 Der pädagogische Aufbau der Erfahrung. Stuttgart: Klett-Cotta.

Radtke, F.-O. (2000). Professionalisierung der Lehrerbildung durch Autonomisierung, Entstaatlichung, Modularisierung. http://www.sowi-online.de/node/1306. Zugegriffen: 16. Aug. 2016.

Reckwitz, A. (2017). Die Gesellschaft der Singularitäten. Frankfurt a. M.: Suhrkamp.

von Rosenberg, F. (2016). Lernen, Bildung kulturelle Pluralität. Auf dem Weg zu einer empirisch fundierten Theorie. Wiesbaden: Springer.

Scheunpflug, A. (2016). Vielfalt Lernen! Eine konstruktive Lernkultur in einer sich globalisierenden Welt. In B. Jehle (Hrsg.), Grundkurs Schulmanagement XIV. Beiträge zur Schul- und Unterrichtsentwicklung - Gestaltungsmöglichkeiten angesichts politisch-gesellschaftlicher Herausforderungen (S. 8-17). Köln: Carl Link.

Schieferdecker, R. (2015). Orientierungen von Lehrerinnen und Lehrern im Themenfeld Heterogenität: Eine rekonstruktive Analyse. Opladen: Barbara Budrich.

Schön, D. A. (1987). Educating the Reflective Practioner. San Francisco: Jossey-Bass.

Timm, S., \& Scheunpflug, A. (2020). Orientierungen im Feld kulturellen Professionshandelns. Empirische Einblicke und Konsequenzen für die Lehrkräftebildung. In S. Timm, J. Costa, C. Kühn \& A. Scheunpflug (Hrsg.), Kulturelle Bildung. Theoretische Perspektiven, methodologische Herausforderungen, empirische Befunde (S. 147-161). Münster: Waxmann.

Vavrus, M. (2008). Culturally responsive teaching. In T.L. Good (Hrsg.), 21st century education: a reference handbook (2. Aufl., S. 49-57). Thousand Oaks: Sage Publishing.

Zacharias, W. (2013). Pluralität und Praxisvielfalt Kultureller Bildung. In Kulturelle Bildung online. https://www.kubi-online.de/artikel/pluralitaet-praxisvielfalt-kultureller-bildung. Zugegriffen: 28. Febr. 2017. 